\title{
Cytokine-release kinetics of platelet-rich plasma according to various activation protocols
}

\section{Y. H. Roh, W. Kim, K. U. Park, J. H. Oh}

Seoul National University College of Medicine, Seongnam, Korea

— Y. H. Roh, MD, PhD, Assistant Professor, Department of Orthopaedic Surgery, Gil Medical Center, Gachon University School of Medicine, Incheon, Korea. n. Wim, MD, Orthopaedic Surgeon, Department of Orthopaedic Surgery, Nalgae Hospital, Seoul, Korea. - K. U. Park, MD, PhD, Professor, Department of Laboratory

Medicine, Seoul National University College of Medicine, Seoul National University Bundang Hospital, Seongnam, Korea. - J. H. Oh, MD, PhD, Professor, Department of Orthopaedic Surgery, Seoul National University College of Medicine, Seongnam, Korea.

Correspondence should be sent to Professor J. H. Oh; e-mail: ohjh1@ snu.ac.kr

doi:10.1302/2046-3758.52.2000540 Bone Joint Res 2016;5:37-45. Received: 17 August 2015; Accepted: 03 December 2015

\section{Objectives}

This study was conducted to evaluate the cytokine-release kinetics of platelet-rich plasma (PRP) according to different activation protocols.

\section{Methods}

Two manual preparation procedures (single-spin (SS) at $900 \mathrm{~g}$ for five minutes; double-spin (DS) at $900 \mathrm{~g}$ for five minutes and then $1500 \mathrm{~g}$ for 15 minutes) were performed for each of 14 healthy subjects. Both preparations were tested for platelet activation by one of three activation protocols: no activation, activation with calcium (Ca) only, or calcium with a low dose (50 IU per $1 \mathrm{mI}$ PRP) of thrombin. Each preparation was divided into four aliquots and incubated for one hour, 24 hours, 72 hours, and seven days. The cytokine-release kinetics were evaluated by assessing PDGF, TGF, VEGF, FGF, IL-1, and MMP-9 concentrations with bead-based sandwich immunoassay.

\section{Results}

The concentration of cytokine released from PRP varied over time and was influenced by various activation protocols. Ca-only activation had a significant effect on the DS PRPs (where the VEGF, FGF, and IL-1 concentrations were sustained) while $\mathrm{Ca}$ /thrombin activation had effects on both SS and DS PRPs (where the PDGF and VEGF concentrations were sustained and the TGF and FGF concentrations were short). The IL-1 content showed a significant increase with $\mathrm{Ca}$-only or $\mathrm{Ca} /$ thrombin activation while these activations did not increase the MMP-9 concentration.

\section{Conclusion}

The SS and DS methods differed in their effect on cytokine release, and this effect varied among the cytokines analysed. In addition, low dose of thrombin/calcium activation increased the overall cytokine release of the PRP preparations over seven days, relative to that with a calcium-only supplement or non-activation.

Cite this article: Bone Joint Res 2016;5:37-45

Keywords: platelet-rich plasma; cytokine; growth factor; kinetics; activation; preparation

\section{Article summary}

Variations in formulations used to activate platelet-rich plasmas (PRPs) result in differences in biological activity of the platelet, which poses methodological challenges to investigators.

- The present study hypothesis is that PRP preparations are different in terms of their quality of cytokine content as a result of the differences in activation protocols.
- PRP preparations can be classified according to preparation protocols as single- or double- spin methods. Single-spin (SS) methods generally result in a lower concentration of platelets and white blood cells (leukocytepoor PRP), and double-spin (DS) methods result in a higher concentration of platelets and white blood cells (leukocyte-rich PRP), so this study evaluates the effects of different activation protocols on cytokine-release kinetics in SS and DS PRP preparations. 


\section{Key messages}

- The PRP preparations showed different qualities in terms of their cytokine-release kinetics depending on activation protocols used.

- The SS and DS methods differed in their effect on cytokine release, and this effect varied among the cytokines analysed.

- A low dose of thrombin/calcium activation increased the overall cytokine release of the PRP preparations over seven days, relative to that with a calcium-only supplement or non-activation.

\section{Strengths and limitations}

- Despite the increase in the use of the PRP for local tissue healing and regeneration in human and animal studies, little is currently known about the biomolecular characteristics of PRP in terms of the cytokinerelease kinetics according to different activation protocols. This study evaluated the cytokine-release kinetics of platelet-rich plasma (PRP) according to different activation protocols.

- Although tissue healing and regeneration generally occur via a multiplicity of cellular interactions, these processes were absent due to the in vitro assay of the current study.

\section{Introduction}

Platelet-rich plasma (PRP) treatments promote tissue healing and regeneration by regulating wound-healing processes and by promoting an anabolic environment at the injury site, 1 and therefore have gained increasing popularity in various areas of orthopaedic surgery. ${ }^{2-7}$ The basis for this treatment has historically centred on maximising the growth factors (GFs) found in platelet alphagranules, such as the platelet-derived growth factor (PDGF), transforming growth factor- $\beta$ (TGF- $\beta$ ), and vascular endothelial growth factor (VEGF). Although evidence of the long-term clinical benefit of PRP has been controversial, 8,9 a large number of studies showed clinical and radiological improvement in patients with muscle or tendon injuries. $2,3,5,10,11$

PRP preparations have been activated from various formulations and have resulted in differences in the biological activity of the platelets, which are sensitive to any type of process-related stress. ${ }^{12-14}$ Inconsistencies in results of clinical or laboratory tests are attributed to sample variability, which poses methodological challenges to investigators. The PRP is clotted to allow for delivery to the desired site, resulting in release of the GFs. Some commercially available systems use thrombin to activate the clotting mechanism, resulting in rapid release of growth factors from the platelets. ${ }^{15}$ An alternative system to delay the release of GF was reported by the addition of calcium chloride, which resulted in formation of a dense fibrin matrix. ${ }^{15}$ Activation with a high dose of thrombin has been reported to induce an immediate release of GFs from platelets, whereas that with a low dose of calcium and thrombin reduces the immediate GF release..$^{16}$

The concentration of the GFs released from PRP varies over time and is influenced by the activation protocols. ${ }^{16,17}$ Degradation of growth factors in vivo can occur via several distinct pathways including denaturation, oxidation or proteolysis. ${ }^{18}$ Most GFs that are contained within platelets have a short life span (minutes to hours). ${ }^{19}$ For instance, the biological half-life of VEGF is less than 30 minutes, while those of PDGF and fibroblast growth factor (FGF) are 2.4 and 7.6 hours, respectively. ${ }^{20-22}$ Thus, some clinical studies have suggested that a repeated injection of PRP improves clinical outcomes. ${ }^{3,23}$ It is therefore crucial to understand the cytokine-release kinetics of PRP in order to determine the practical methods of the PRP application, such as the dose and the number of PRP injections. Several studies have demonstrated differences in both the platelet and cytokine concentrations in PRP systems, ${ }^{14,24,25}$ but these have only focused on comparing the platelet or cytokine concentration after an uncertain incubation period. The effect of different activation protocols on cytokine-release kinetics of PRP preparations has not been evaluated in depth.

The purpose of this study was to evaluate the cytokinerelease kinetics of PRP according to different activation protocols. The present study hypothesis is that PRP preparations are different in terms of their quality of cytokine content as a result of the differences in activation protocols. PRP preparations can be classified according to preparation protocols as single- or double-spin methods. ${ }^{14}$ Single-spin (SS) methods generally result in a lower concentration of platelets and white blood cells (leukocytepoor PRP), and double-spin (DS) methods result in a higher concentration of platelets and white blood cells (leukocyte-rich PRP), so this study evaluates the effects of different activation protocols: non-activation, Ca-only and $\mathrm{Ca}$ /thrombin activation, on cytokine-release kinetics in SS and DS PRP preparations.

\section{Materials and Methods}

Subjects. Blood samples were obtained from 14 healthy subjects (seven females, seven males; mean age 34.6 years, standard deviation (SD) $6.6 ; 26$ to 50 ). This study was approved by our institutional review board, and all patients signed an informed consent form. The inclusion criteria included healthy subjects between the ages of 18 and 65 years without any known blood dyscrasia, and the exclusion criteria included medical history of any blood-derived illness or medication known to affect platelet or bone marrow function for a minimum of two weeks prior to testing.

Platelet-rich plasma preparation. Peripheral venous blood was collected into acid-citrate dextrose A (ACD-A) anticoagulant with a ratio of $1 \mathrm{ml} \mathrm{ACD}-A$ to $9 \mathrm{~mL}$ of 
Table I. Preparation protocols and cellular compositions of PRP preparations.

\begin{tabular}{|c|c|c|c|c|c|c|c|}
\hline Preparations & $\begin{array}{l}\text { Centrifugations } \\
\text { First spin }\end{array}$ & Second spin & Isolation & $\begin{array}{l}\text { Final vol./ } \\
\text { WB vol. }\end{array}$ & $\begin{array}{l}\text { Platelet } \\
\left(10^{3} \text { cells/uL) }\right.\end{array}$ & $\begin{array}{l}\text { WBC } \\
\left(10^{3} \text { cells/uL) }\right.\end{array}$ & $\begin{array}{l}\text { RBC } \\
\left(10^{3} \text { cells } / u L\right)\end{array}$ \\
\hline Control (whole-blood) & & & & $3 \mathrm{~mL}$ & 147 (SD 59) & $6.27($ SD 1.94) & 4269 (SD 393) \\
\hline SS preparation & $900 \mathrm{~g}-5 \mathrm{~min}$ & & Plasma layer & $3 \mathrm{~mL} / 30 \mathrm{~mL}$ & 311 (SD 72) & $0.28(S D 0.14)$ & $13($ SD 6$)$ \\
\hline DS preparation & $900 \mathrm{~g}-5 \mathrm{~min}$ & $1500 \mathrm{~g}-15 \mathrm{~min}$ & Plasma layer & $3 \mathrm{~mL} / 30 \mathrm{~mL}$ & 1145 (SD 244) & $2.63($ SD 1.54) & 70 (SD 34) \\
\hline
\end{tabular}

SS, single spin method; DS, double spin method; WBC, white blood cell; RBC, red blood cell; SD, standard deviation

whole blood. The optimal separating centrifugation condition (first spin) has been suggested to be $900 \mathrm{~g}$ for five minutes with a platelet recovery ratio (the total platelet counts after centrifugation divided by those before centrifugation) of $92.0+3.1 \%$, and the optimal condensation centrifugation condition (second spin) is $1500 \mathrm{~g}$ for 15 minutes with a recovery ratio of $84.3+10.0 \% .^{26}$ In this study, the same protocols were used for the SS and the DS PRP preparations. For the SS PRP preparation, $30 \mathrm{ml}$ of whole blood was centrifuged at $900 \mathrm{~g}$ for five minutes. Then the top plasma layer was separated, and the lower $3 \mathrm{ml}$ of volume of the plasma were used as the SS PRP preparation. (The lower cell layer, which contained leukocytes and erythrocytes, was removed in this process and thus, the upper plasma layer presented a decrease in the concentration of leukocyte and erythrocyte.) For the DS PRP preparation, the top plasma layer after the single spin was separated and was centrifuged a second time under $1500 \mathrm{~g}$ for 15 minutes, and the superficial plasma layer was removed, and finally, the lower $3 \mathrm{ml}$ of volume of the plasma were used as the DS PRP preparation.

SS/DS PRP activation. Three different conditions were tested for exogenous activation: no activation, calcium gluconate only, and calcium and thrombin (Dede R Thrombin reagent, Siemens, Germany). Calcium gluconate $(2.084 \mathrm{~g} / 20 \mathrm{ml})$ was added to PRP at a ratio of $1: 10$, and thrombin was added at $50 \mathrm{IU}$ per $1 \mathrm{ml}$ volume of PRP, which is less than that reported in some previous studies, ${ }^{27-31}$ to prevent any early depletion of GFs and to induce a sustained cytokine release over seven days. Calcium has a role in platelet aggregation and structure of platelet surface receptors and subsequently the function of these receptors is modified by the presence of calcium. ${ }^{32,33}$ However, ACD-A is a potent calcium chelator and it may block activation of thrombin. Therefore, adding calcium in thrombin activation was considered more physiological than thrombin activation alone.

Evaluation of the cellular composition and kinetics of cytokine release. The platelet, white blood cell (WBC), and red blood cell (RBC) counts were performed before cytokine quantification by using a cell count machine (XE2100, Sysmex, Mundelein, Illinois). Most haematology analysers are designed to operate within ranges found in whole blood, and PRP may exceed the upper limit of the linear range of platelets that can be counted. Therefore, PRP was suspended with normal saline at a ratio of 1:5, and the cellular content of PRP was analysed.
Each PRP preparation or whole blood (control) sample was divided into four aliquots and was incubated for one hour, 24 hours, 72 hours and seven days at $37^{\circ} \mathrm{C}$ in $5 \%$ $\mathrm{CO}_{2}$. The samples were snap-frozen in individual aliquots after a given incubation period and were stored at $-80^{\circ} \mathrm{C}$ for a cytokine assay. These samples were then assayed in duplicate, and platelet-derived growth factor -BB (PDGF-BB), transforming growth factor- $\beta 1$ (TGF- $\beta 1$ ), vascular endothelial growth factor (VEGF), basic fibroblast growth factor (bFGF), interleukin-1 $\beta$ (IL-1 $\beta$ ), and matrix metalloproteinase-9 (MMP-9) concentrations were evaluated using commercially available bead-based sandwich immune-assay kits (Bio-Rad Laboratories, Hercules, California).

Previous studies have demonstrated that PRP concentrates platelets, and therefore the GFs, contained in alpha granules. $4,14,15$ PDGF, TGF- $\beta$, VEGF, and FGF, which play a crucial role in cell proliferation and differentiation in the wound-healing process, ${ }^{17}$ are basic cytokines identified in platelets and were chosen for anabolic cytokines to be analysed. These growth factors are also considered the most important growth factors in tendon healing. ${ }^{34}$ Conversely, effects of PRP are not only attributable to concentrated platelets but also to the presence of leukocyte and catabolic cytokines. ${ }^{25}$ Thus, IL-1 $\beta$ and MMP-9 were chosen for catabolic cytokines, which play roles in inflammation or matrix degradation. ${ }^{25}$

Statistical analysis. The measured data are presented as the arithmetic mean and the standard deviation (SD). The concentrations of the cytokines in each of the incubation periods were analysed using a Kruskal-Wallis test (non-parametric ANOVA), a Bonferroni posthoc test was conducted to compare multiple values from each of the preparation conditions, and linear correlations between the cell count and cytokine content were analysed via Pearson correlations. A p-value of 0.05 was considered significant.

\section{Results}

The mean platelet concentration of the control samples was $147 \times 10^{3} / \mathrm{uL}$ (SD 59). The platelet concentration of the SS PRP at $311 \times 10^{3} / \mathrm{uL}$ (SD 72) is significantly higher than that of the control $(p<0.01)$, and that of the DS PRP is even higher at $1145 \times 10^{3} / u L(S D 244)(p<0.01)$. The WBC concentration of the DS PRP was about nine times higher than that of the SS PRP $(p<0.01)$, but both concentrations were lower than those of the control samples (Table I). 
Time-sequential cytokine release

PDGF-BB. The PDGF-BB release was constant and was sustained over seven days. The PDGF-BB concentrations of PRP were correlated with platelet concentrations ( $r=0.71, p=0.02)$, and the PDGF concentrations of the DS PRP were two to three times higher than those of the SS PRP over a seven-day period $(p<0.01$ for all concentrations, Fig. 1a). Ca-only activation did not significantly increase the PDGF-BB concentration for either the SS or the DS PRP preparations (Fig. 1b), whereas Ca/ thrombin activation induced an immediate and sustainable increase in the PDGF-BB concentration for both SS and DS PRPs over the seven-day period ( $p<0.01$ for all measured times, Fig. 1c).

TGF- $\beta 1$. The TGF- $\beta 1$ release was rapidly induced, and a maximum concentration was detected within one hour with no significant differences in the TGF- $\beta 1$ concentration between the SS and DS PRP preparations over the seven-day period (Fig. 2a). The TGF- $\beta 1$ concentration was not correlated with the platelet concentration of the PRP $(r=0.18, p=0.32)$. Ca-only activation did not increase the TGF- $\beta 1$ concentration of the SS or the DS PRP (Fig. 2b). $\mathrm{Ca} /$ thrombin activation immediately, but not sustainably, increased the TGF- $\beta 1$ concentration of SS PRP at one hour and 24 hours ( $p<0.01$ and 0.02 , respectively) and that of DS PRP at one hour and 24 hours $(p<0.01$ and 0.02 , respectively, Fig. 2c).

VEGF. The VEGF concentration of the PRP was correlated with the platelet concentration $(r=0.71, p=0.02)$ and was constant and sustained over seven days. The VEGF concentration of the DS PRP exceeded that of SS PRP at 72 hours and after seven days $(p=0.06$ and $<0.01$, respectively, Fig. 3a). The Ca activation immediately and sustainably increased the VEGF concentration of the DS PRP at all measured times ( $p<0.01$ for all measured times, Fig. $3 b$ ). The $\mathrm{Ca}$ /thrombin activation sustainably increased the VEGF concentration of DS PRP $(p<0.01$, $<0.01,<0.01,0.04$ at one hour, 24 hours, 72 hours, and seven days, respectively) but increased that of SS PRP only at one hour and 24 hours $(p<0.01$ and 0.02 , respectively, Fig. 3c).

FGF. The FGF release was rapidly induced and a maximum concentration was detected within one hour, but the FGF concentration was not correlated with the platelet concentration $(r=0.21, p=0.26)$. The FGF concentration of the SS PRP exceeded that of DS PRP at 24 hours, 72 hours, and seven days ( $p \leq 0.01,<0.01$, and 0.04, respectively, Fig. 4a). The Ca-only activation increased the FGF concentration of DS PRP at 24 hours, 72 hours and seven days $(p<0.01,<0.01$, and 0.03 , respectively), but reduced that of the SS PRP at all measured times $(p<0.01$ for all measured times, Fig. $4 b$ ). The $\mathrm{Ca} /$ thrombin activation immediately, but not sustainably, increased the FGF concentration of SS PRP $(p<0.01$ at one hour $)$ and that of DS PRP ( $p=0.01$ and $<0.01$, at one and 24 hours, respectively, Fig. 4c).
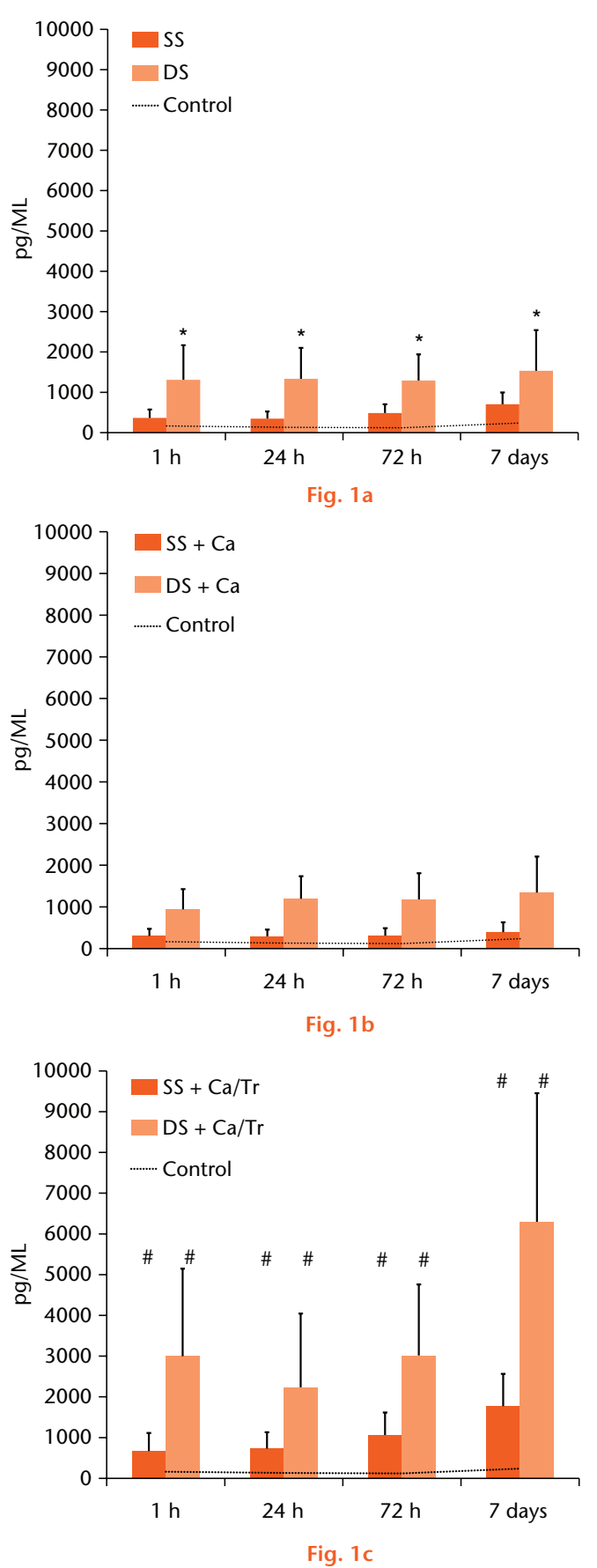

a) The PDGF-BB release was sustained over seven days. b) Ca-only activation did not significantly increase the PDGF-BB concentration for either the SS or the DS PRP preparations, c) whereas $\mathrm{Ca}$ /thrombin activation induced an immediate and sustainable increase in the PDGF-BB concentration for both SS and DS PRPs over the seven-day period. (* represents $p<0.05$ compared with the SS group, \# represents $p<0.05$ compared with the non-activation group.)

IL-1 1 . IL-1 $\beta$ release was constant and was sustained over seven days. There were no significant differences in the IL-1 $\beta$ concentration between the SS and DS PRP preparations (Fig. 5a). The IL-1 $\beta$ concentration was not correlated with the WBC or the PLT concentration $(r=0.19$, $p=0.30$ ), and the Ca activation led to a ten- to 20 -fold increase in IL-1 $\beta$ concentration for DS PRP at 24 hours, 72 hours, and seven days $(p=0.02,<0.01$, and $<0.01$, 

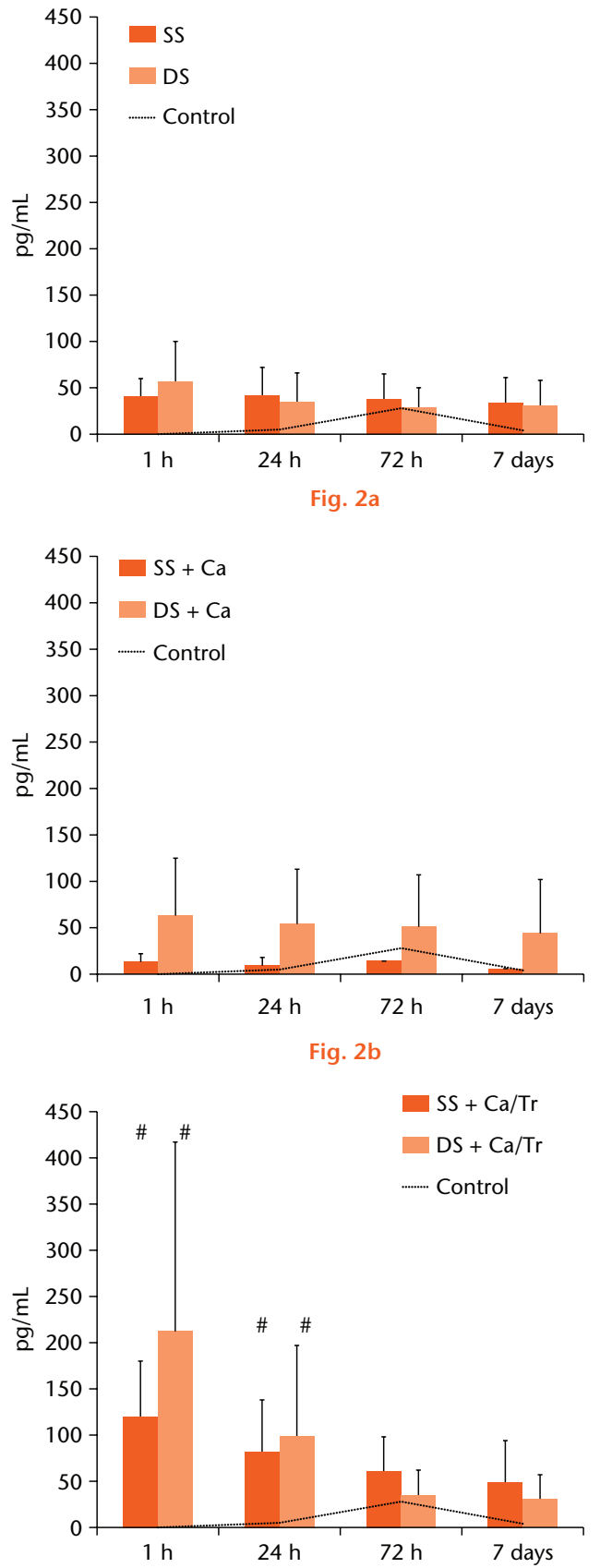

Fig. 2c

a) The TGF- $\beta 1$ release was rapidly induced, and a maximum concentration was detected within one hour. b) Ca-only activation did not increase the TGF- $\beta 1$ concentration of the SS or the DS PRP, and c) Ca/thrombin activation immediately, but not sustainably, increased the TGF- $\beta 1$ concentration of SS PRP at one hour and 24 hours and that of DS PRP at one hour and 24 hours. (* represents $p<0.05$ compared with the SS group, \# represents $p<0.05$ compared with the non-activation group.)

respectively, Fig. $5 b)$. The $\mathrm{Ca} /$ thrombin activation led to a two- to ten-fold increase in this concentration for the SS PRP over seven days ( $p<0.01$ for all measured times, Fig. 5C).

MMP-9. The MMP-9 concentration was lower than $50 \mathrm{pg} / \mathrm{mL}$ over seven days for both the SS and DS PRP preparations, and these values were lower than that of
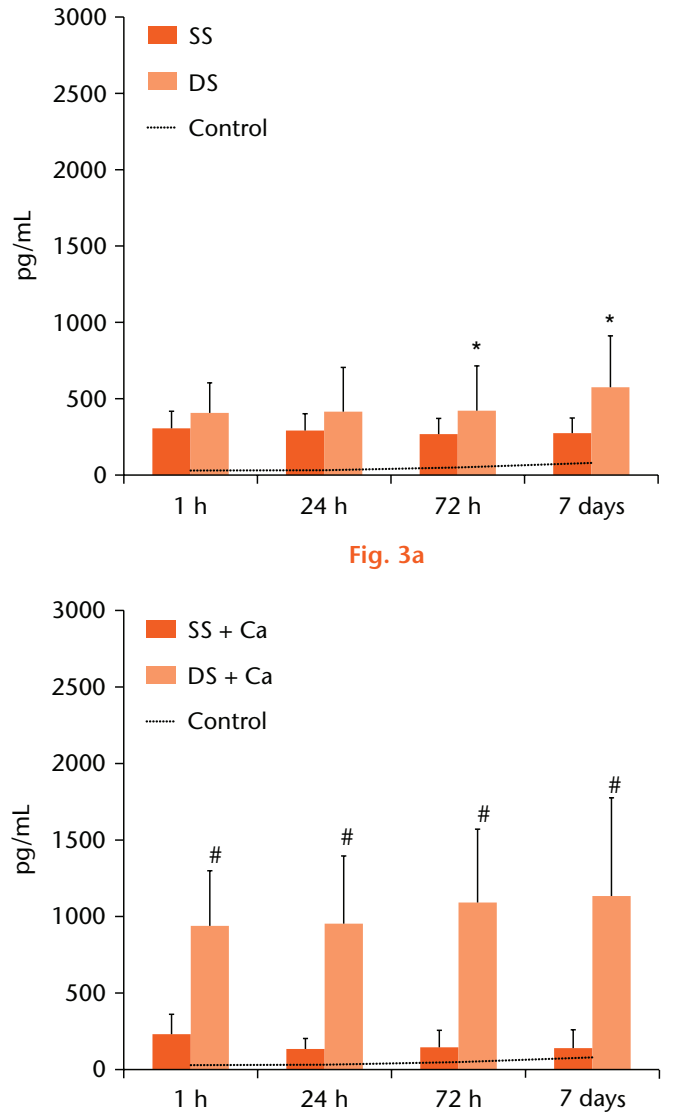

Fig. $3 b$

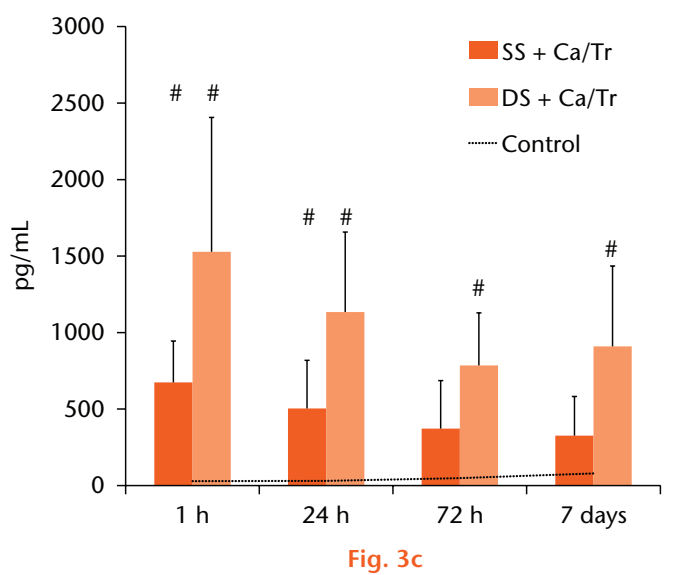

a) The VEGF release was constant and sustained over seven days. b) The Ca activation immediately and sustainably increased the VEGF concentration of the DS PRP at all measured times. c) The $\mathrm{Ca}$ /thrombin activation sustainably increased the VEGF concentration of DS PRP, but increased that of SS PRP only at one hour and 24 hours. (* represents $p<0.05$ compared with the SS group, \# represents $p<0.05$ compared with the non-activation group.)

control (Fig. 6a). The MMP-9 concentration was strongly correlated with the WBC concentration $(r=938, p<01)$. There were no significant differences in the MMP-9 concentration between the SS and the DS PRP preparations. Ca-only or $\mathrm{Ca}$ /thrombin activation did not increase the MMP-9 concentration for either the SS or the DS PRP preparations (Figs $6 \mathrm{~b}$ and $6 \mathrm{c}$ ). 

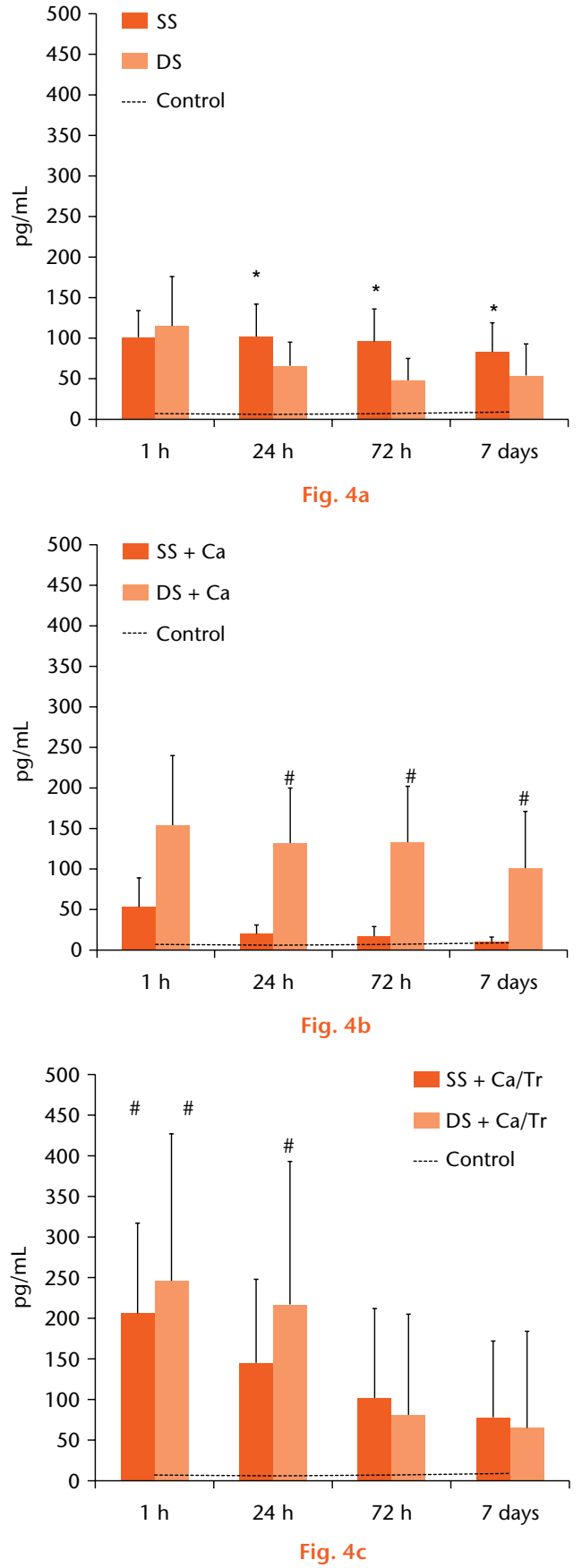

a) The FGF release was rapidly induced and a maximum concentration was detected within one hour. b) The Ca-only activation increased the FGF concentration of DS PRP at 24 hours, 72 hours and seven days. c) The $\mathrm{Ca}$ /thrombin activation immediately, but not sustainably, increased the FGF concentration of SS PRP and that of DS PRP. (* represents $p<0.05$ compared with the DS group, \# represents $p<0.05$ compared with the non-activation group.)

\section{Discussion}

Despite the increase in the use of the PRP for local tissue healing and regeneration in human and animal studies, little is currently known about the biomolecular characteristics of PRP in terms of the cytokine-release kinetics according to different activation protocols. It is important to determine the method of local application as
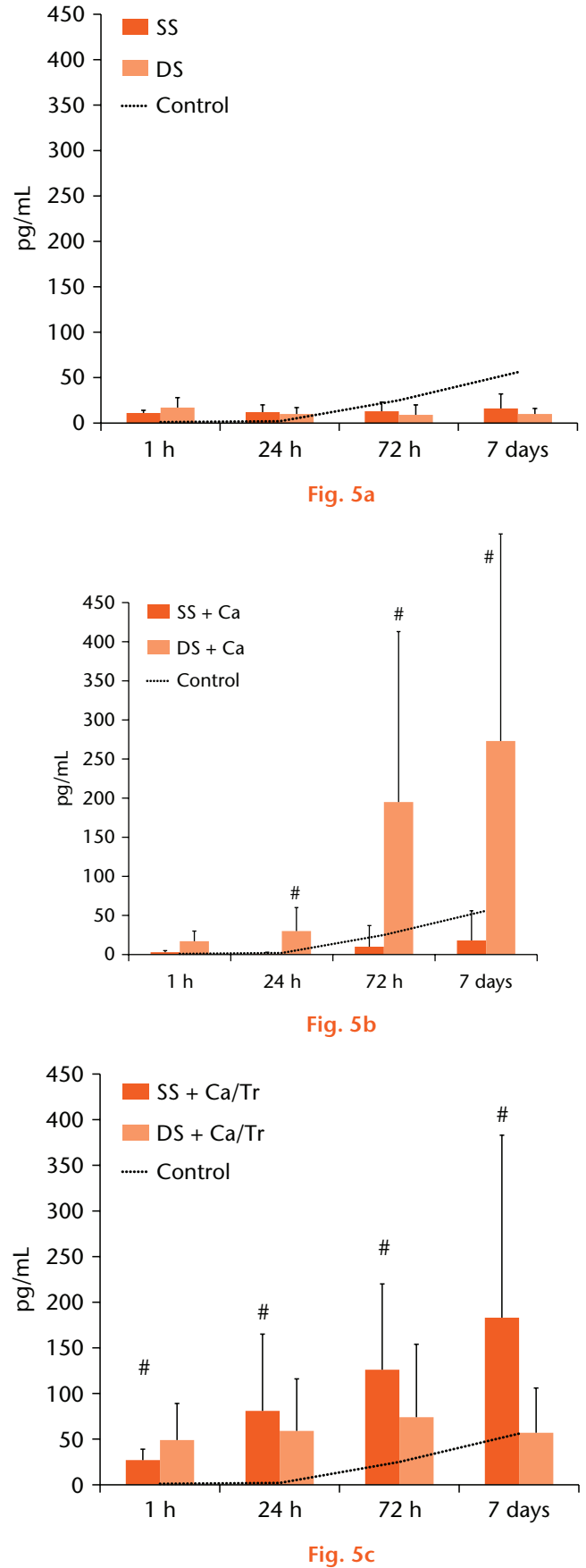

a) There were no significant differences in the IL-1 $\beta$ concentration between the SS and DS PRP preparations. b) The Ca activation led to a ten- to 20-fold increase in IL-1 $\beta$ concentration for the DS PRP at 24 hours, 72 hours, and seven days. c) The $\mathrm{Ca}$ /thrombin activation led to a two- to ten-fold increase in this concentration for the SS PRP over seven days. (* represents $p<0.05$ compared with the SS group, \# represents $\mathrm{p}<0.05$ compared with the non-activation group.)

well as to evaluate the effectiveness of the procedure. The concentration of cytokines released from PRP varied over time and was influenced by various activation protocols. The effect of the activation was shown to be dependent on the preparation method as well as on the type of cytokine and, accordingly, proper PRP components with activation methods should be selected by 

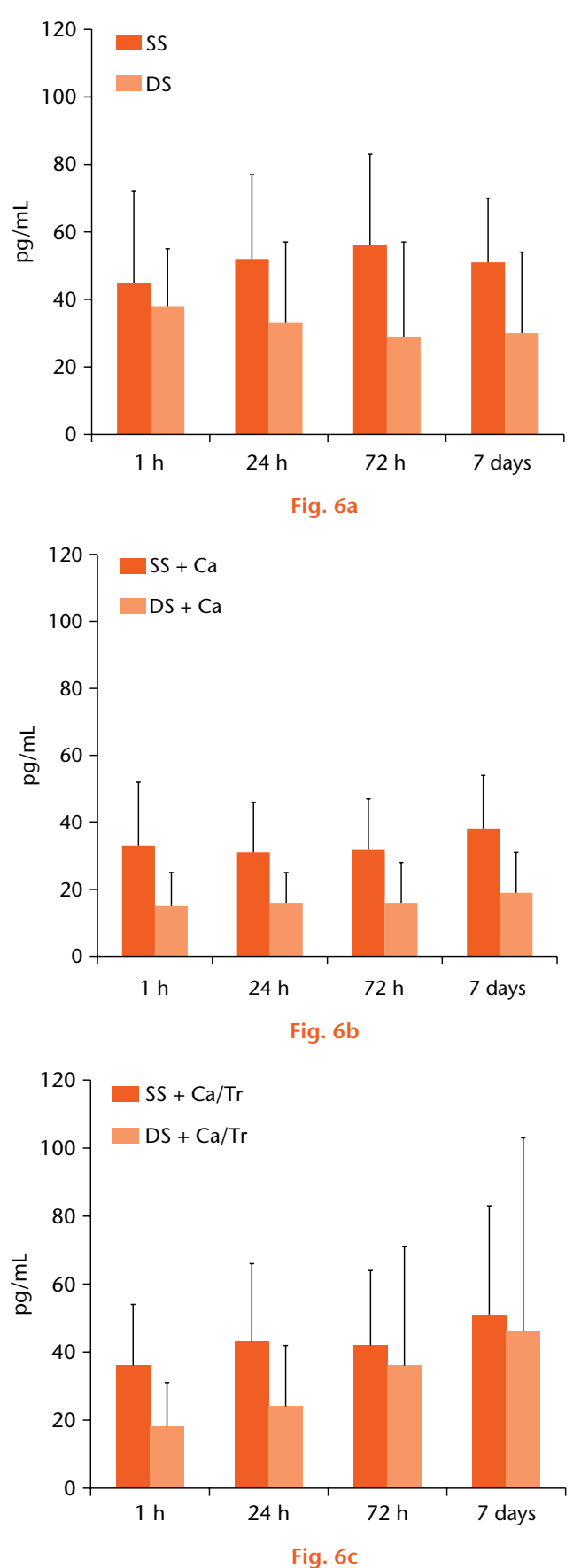

a) The MMP-9 concentration for control samples (whole blood) was greater than $500 \mathrm{pg} / \mathrm{mL}$ over seven days. There were no significant differences in the MMP-9 concentration between the SS and the DS PRP preparations. b, c) Caonly or $\mathrm{Ca}$ /thrombin activation did not increase the MMP-9 concentration for either the SS or the DS PRP preparations. (* represents $p<0.05$ compared with the SS group, \# represents $p<0.05$ compared with the non-activation group).

considering their biomolecular characteristics and patient indications.

The PDGF-BB, TGF- $\beta 1$, VEGF, and FGF concentrations in PRP are known to play a crucial role in cell proliferation, chemotaxis, cell differentiation, and angiogenesis. ${ }^{35}$ PDGF is a powerful mitogen for fibroblasts and smooth muscle cells. ${ }^{36}$ TGF- $\beta$ stimulates the proliferation of undifferentiated mesenchymal stem cells and the chemotaxis of endothelial cells and angiogenesis. ${ }^{37}$ VEGF stimulates endothelial cell mitogenesis and cell migration, ${ }^{38}$ and FGF plays a key role in the proliferation and differentiation of a wide variety of cells and tissues. ${ }^{39}$ Conversely, IL-1 $\beta$ and MMP-9 are catabolic cytokines that are known to play roles in inflammation or matrix degradation. ${ }^{25}$ Interleukin- $1 \beta$ is a primary cytokine during inflammation and matrix degradation, and it is a common target to reduce inflammation by manipulating IL-1 ra. ${ }^{40}$ MMP-9 is known to degrade collagen and other extracellular matrix molecules and has been implicated as a predictor of poor healing. 41

The activation method or the thrombin dose affects the cytokine-release kinetics of PRPs and must be considered when interpreting the results of PRP studies. When calcium chloride was added exogenously to the PRP, a low level of thrombin formed endogenously and allowed a slower GF release over a longer period than exogenous thrombin. On the other hand, thrombin caused a rapid aggregation of platelets and an excessive condensing of the fibrin matrix with a rapid activation of the platelets. A low dose of thrombin has been shown to increase the migration and the number of mesenchymal progenitor cells derived from bone marrow $^{42}$ whereas high concentrations have been demonstrated to have limited effects on the proliferation of osteoblasts and alveolar bone cell, suggesting that the thrombin dose plays a role in the GF-release kinetics of the PRP preparations. ${ }^{43}$

The effect of the activation depended on both the preparation method and on the type of cytokine assessed. Ca-only activation had a significant effect on the DS PRP preparation (VEGF, FGF, and IL-1 $\beta$ concentrations) whereas $\mathrm{Ca}$ /thrombin activation had significant effects on both SS and DS PRP preparations (PDGF-BB and VEGF concentrations sustainably and TGF and FGF concentrations shortly). These observations may be due to the fact that the biological activity of the platelets is sensitive to any kind of process-related stress and that more platelets are activated during the process with the DS method. These results are also consistent with the findings reported by Mazzucco et al, ${ }^{13}$ who reported that the individual dynamics of the GF release depend exclusively on the type of GF rather than on the preparation method. They also demonstrated that TGF- $\beta 1$ and bFGF are promptly released within 24 hours of exogenous activation whereas the GF release of the PDGF-BB and the VEGF are more dependent on the technique that is used.

In terms of the catabolic cytokines, Ca or thrombin activation sustainably increased the IL-1 $\beta$ concentration to a level ten times higher than that of the control but did not increase the MMP-9 release over time. These results suggest that the mechanisms underlying the synthesis, release, and/or degradation of IL-1 $\beta$ differ from those of MMP-9 and that the release of IL-1 $\beta$ may be a result of some 
de novo synthesis by leukocytes or platelets following PRP activation with a low concentration of $\mathrm{Ca}$ or thrombin. ${ }^{44}$

This study has several limitations. Our experimental set up only measured absolute cytokine levels (accumulation over time) in solution at the time points and did not account for cytokine depletion or breakdown over time. Degradation of growth factors in vivo can occur via several distinct pathways including denaturation, oxidation or proteolysis, which were not measured in the present study. Second, although tissue healing and regeneration generally occur via a multiplicity of cellular interactions, these processes were absent due to the in vitro nature of the current study. Further studies may be needed to investigate the relationship between the GF kinetics and tissue healing/regeneration in vivo. Third, the cytokine content is influenced mainly by inter-individual variability, which in turn may influence the release kinetics of the PRP GFs. Therefore, there may be some differences in their characteristics for other populations. Finally, not all PRP kits or activation protocols could be considered due to patient recruitment and financial limitations. Although we considered the preparation and activation methods used in this study as representative techniques, various other methods are also commercially available for the preparation of PRP. These protocols can differ in centrifuge conditions and processes of activation.

PRP has distinct characteristics that reflect specific mixtures of bioactive molecules, and the regenerative potency of PRP may depend on the GF levels. Cytokine content was observed to be different between the SS and DS methods. A low dose of thrombin/calcium activation caused an overall increase in cytokine content over a period of seven days when compared with a calcium-only supplement or non-activation preparations, and the effect of the activation depended not only on the preparation method but also on the type of cytokine. Ca-only activation had a significant effect on DS PRP preparation while a low dose of thrombin with calcium activation had significant effects on both SS and DS PRP preparations. Low dose of thrombin activation is recommended for enhancing growth factor contents of PRPs. Physicians and researchers should interpret results of clinical or laboratory tests of PRP in the context of their activation protocols.

\section{References}

1. Werner S, Grose R. Regulation of wound healing by growth factors and cytokines. Physiol Rev 2003;83:835-870.

2. Sanli I, Morgan B, van Tilborg F, Funk L, Gosens T. Single injection of plateletrich plasma (PRP) for the treatment of refractory distal biceps tendonitis: long-term results of a prospective multicenter cohort study. Knee Surg Sports Traumatol Arthrosc 2014. [Epub ahead of print]

3. Charousset C, Zaoui A, Bellaiche L, Bouyer B. Are multiple platelet-rich plasma injections useful for treatment of chronic patellar tendinopathy in athletes? a prospective study. Am J Sports Med 2014;42:906-911.

4. Hsu WK, Mishra A, Rodeo SR, et al. Platelet-rich plasma in orthopaedic applications: evidence-based recommendations for treatment. J Am Acad Orthop Surg 2013;21:739-748.
5. Mishra AK, Skrepnik NV, Edwards SG, et al. Efficacy of platelet-rich plasma for chronic tennis elbow: a double-blind, prospective, multicenter, randomized controlled trial of 230 patients. Am J Sports Med 2014;42:463-471.

6. Jo CH, Kim JE, Yoon KS, Shin S. Platelet-rich plasma stimulates cell proliferation and enhances matrix gene expression and synthesis in tenocytes from human rotator cuff tendons with degenerative tears. Am J Sports Med 2012;40:1035-1045.

7. Duif C, Vogel T, Topcuoglu F, et al. Does intraoperative application of leukocytepoor platelet-rich plasma during arthroscopy for knee degeneration affect postoperative pain, function and quality of life? A 12-month randomized controlled double-blind trial. Arch Orthop Trauma Surg 2015;135:971-977.

8. Silva A, Sampaio R. Anatomic ACL reconstruction: does the platelet-rich plasma accelerate tendon healing? Knee Surg Sports Traumatol Arthrosc 2009;17:676-682.

9. Rodeo SA, Delos D, Williams RJ, et al. The effect of platelet-rich fibrin matrix on rotator cuff tendon healing: a prospective, randomized clinical study. Am J Sports Med 2012;40:1234-1241.

10. Gosens T, Peerbooms JC, van Laar W, den Oudsten BL. Ongoing positive effect of platelet-rich plasma versus corticosteroid injection in lateral epicondylitis: a double- blind randomized controlled trial with 2-year follow-up. Am J Sports Med 2011;39:1200-1208.

11. Randelli P, Arrigoni P, Ragone V, Aliprandi A, Cabitza P. Platelet rich plasma in arthroscopic rotator cuff repair: a prospective RCT study, 2-year follow-up. J Shoulder Elbow Surg 2011;20:518-528.

12. Cavallo C, Filardo G, Mariani E, et al. Comparison of platelet-rich plasma formulations for cartilage healing: an in vitro study. J Bone Joint Surg [Am] 2014; 96-A:423-429.

13. Mazzucco L, Balbo V, Cattana E, Guaschino R, Borzini P. Not every PRP-gel is born equal. Evaluation of growth factor availability for tissues through four PRP-gel preparations: Fibrinet, RegenPRP-Kit, Plateltex and one manual procedure. Vox Sang 2009;97:110-118

14. Mazzocca AD, McCarthy MB, Chowaniec DM, et al. Platelet-rich plasma differs according to preparation method and human variability. J Bone Joint Surg [Am] 2012:94-A:308-316.

15. Foster TE, Puskas BL, Mandelbaum BR, Gerhardt MB, Rodeo SA. Platelet-rich plasma: from basic science to clinical applications. Am J Sports Med 2009;37:2259-2272.

16. Lacoste E, Martineau I, Gagnon G. Platelet concentrates: effects of calcium and thrombin on endothelial cell proliferation and growth factor release. J Periodontol 2003;74:1498-1507.

17. Roussy Y, Bertrand Duchesne MP, Gagnon G. Activation of human platelet-rich plasmas: effect on growth factors release, cell division and in vivo bone formation. Clin Oral Implants Res 2007;18:639-648.

18. Lee K, Silva EA, Mooney DJ. Growth factor delivery-based tissue engineering general approaches and a review of recent developments. J R Soc Interface 2011:8:153-170.

19. Isaac C, Gharaibeh B, Witt M, Wright VJ, Huard J. Biologic approaches to enhance rotator cuff healing after injury. J Shoulder Elbow Surg 2012;21:181-190.

20. Gay CG, Winkles JA. The half-lives of platelet-derived growth factor A- and B-chain mRNAs are similar in endothelial cells and unaffected by heparin-binding growth factor-1 or cycloheximide. J Cell Physiol 1991;147:121-127.

21. Beenken A, Mohammadi M. The FGF family: biology, pathophysiology and therapy. Nat Rev Drug Discov 2009;8:235-253.

22. Eppler SM, Combs DL, Henry TD, et al. A target-mediated model to describe the pharmacokinetics and hemodynamic effects of recombinant human vascular endothelial growth factor in humans. Clin Pharmacol Ther 2002;72:20-32.

23. Gobbi A, Lad D, Karnatzikos G. The effects of repeated intra-articular PRP injections on clinical outcomes of early osteoarthritis of the knee. Knee Surg Sports Traumatol Arthrosc 2015;23:2170-2177.

24. Magalon J, Bausset $\mathbf{0}$, Serratrice $\mathbf{N}$, et al. Characterization and comparison of 5 platelet-rich plasma preparations in a single-donor model. Arthroscopy 2014;30:629-638.

25. Sundman EA, Cole BJ, Fortier LA. Growth factor and catabolic cytokine concentrations are influenced by the cellular composition of platelet-rich plasma. Am J Sports Med 2011;39:2135-2140

26. Jo CH, Roh YH, Kim JE, Shin S, Yoon KS. Optimizing platelet-rich plasma gel formation by varying time and gravitational forces during centrifugation. J Oral Implantol 2013;39:525-532.

27. Martineau I, Lacoste E, Gagnon G. Effects of calcium and thrombin on growth factor release from platelet concentrates: kinetics and regulation of endothelial cell proliferation. Biomaterials 2004;25:4489-4502.

28. Marx RE, Carlson ER, Eichstaedt RM, et al. Platelet-rich plasma: growth factor enhancement for bone grafts. Oral Surg Oral Med Oral Pathol Oral Radiol Endod 1998:85:638-646. 
29. Berghoff WJ, Pietrzak WS, Rhodes RD. Platelet-rich plasma application during closure following total knee arthroplasty. Orthopedics 2006;29:590-598.

30. Han B, Woodell-May J, Ponticiello M, Yang Z, Nimni M. The effect of thrombin activation of platelet-rich plasma on demineralized bone matrix osteoinductivity. J Bone Joint Surg [Am] 2009;91-A:1459-1470.

31. Scherer SS, Tobalem M, Vigato E, et al. Nonactivated versus thrombin-activated platelets on wound healing and fibroblast-to-myofibroblast differentiation in vivo and in vitro. Plast Reconstr Surg 2012;129:46e-54e.

32. Nesbitt WS, Giuliano S, Kulkarni S, et al. Intercellular calcium communication regulates platelet aggregation and thrombus growth. J Cell Bio/ 2003;160:1151-1161.

33. Dionisio N, Redondo PC, Jardin I, Rosado JA. Transient receptor potential channels in human platelets: expression and functional role. Curr Mol Med 2012;12:1319-1328.

34. Müller SA, Todorov A, Heisterbach PE, Martin I, Majewski M. Tendon healing: an overview of physiology, biology, and pathology of tendon healing and systematic review of state of the art in tendon bioengineering. Knee Surg Sports Traumatol Arthrosc 2015;23:2097-2105.

35. Sanchez-Gonzalez DJ, Mendez-Bolaina E, Trejo-Bahena NI. Platelet-rich plasma peptides: key for regeneration. Int J Pept 2012;2012:532519.

36. Hosgood G. Wound healing. The role of platelet-derived growth factor and transforming growth factor beta. Vet Surg 1993;22:490-495.

37. Civinini R, Nistri L, Martini C, et al. Growth factors in the treatment of early osteoarthritis. Clin Cases Miner Bone Metab 2013;10:26-29.

38. Hoeben A, Landuyt B, Highley MS, et al. Vascular endothelial growth factor and angiogenesis. Pharmacol Rev 2004;56:549-580.

39. Cao R, Brakenhielm E, Pawliuk R, et al. Angiogenic synergism, vascular stability and improvement of hind-limb ischemia by a combination of PDGF-BB and FGF-2. Nat Med 2003:9:604-613.
40. Thampatty BP, Li H, Im HJ, Wang JH. EP4 receptor regulates collagen type-I, MMP-1, and MMP-3 gene expression in human tendon fibroblasts in response to ILbeta treatment. Gene 2007;386:154-161.

41. Chakraborti S, Mandal M, Das S, Mandal A, Chakraborti T. Regulation of matrix metalloproteinases: an overview. Mol Cell Biochem 2003;253:269-285.

42. Gruber R, Karreth F, Kandler B, et al. Platelet-released supernatants increase migration and proliferation, and decrease osteogenic differentiation of bone marrowderived mesenchymal progenitor cells under in vitro conditions. Platelets 2004;15:29-35.

43. Choi BH, Zhu SJ, Kim BY, et al. Effect of platelet-rich plasma (PRP) concentration on the viability and proliferation of alveolar bone cells: an in vitro study. Int J Oral Maxillofac Surg 2005:34:420-424.

44. Frechette JP, Martineau I, Gagnon G. Platelet-rich plasmas: growth factor content and roles in wound healing. J Dent Res 2005;84:434-439.

Funding Statement

This study was funded by the Seoul National University Bundang Hospital's research fund (No. 02-2012-012).

Author Contributions

Y. H. Roh, Study design, Data analysis, Writing the paper.

W. Kim, Data collection, Data analysis.

K. U. Park, Data collection, Data analysis.

J. H. Oh, Study design, Revising the paper.

ICMJE conflict of interest

None declared.

() 2016 Oh et al. This is an open-access article distributed under the terms of the Creative Commons Attributions licence (CC-BY-NC), which permits unrestricted use, distribution, and reproduction in any medium, but not for commercial gain, provided the original author and source are credited. 